\title{
Structured Query Language (SQL) Answering Model for User Queries based on Intuitionistic Fuzzy Logic
}

\author{
Ashit Kumar Dutta, PhD \\ Associate Professor \\ Shaqra University
}

\begin{abstract}
Instuitionistic fuzzy logic is widely accepted method to analyse the imprecise and vague data. There are number of database management systems (DBMS) are available to facilitate the users to store and organize the data for the future purpose. DBMS lacks to understand the user queries in distributed environment. Sql is a popular querying language to fetch data depend upon the user queries. The structure of the sql query is designed for precise queries from the user but it will return error for vague queries. As the business extends from one part of the world to the other language should be a barrier for the non - English speakers. The research is to find the solution for the problems exist in the sql to translate the user queries. The proposed model will answer all kind of user queries and tries solve the vagueness problem using fuzzy logic.
\end{abstract}

\section{General Terms}

Intutionistic fuzzy, Sql, Distributed Database, Query optimization.

\section{Keywords}

Answering system, Fuzzifier, Translator

\section{INTRODUCTION}

Database is the nervous system of software. If database is not properly organized then whole software will be paralyzed and consumes lot of time to set right the whole system. [1][2][3]Distributed database is one of the prime technology for the software industry to use their application all over the world.[6][7] It increase the speed of the application by employing multiple site server accessibility to the user. Internet, intranet and extranet are the port of distributed database applications. Structured query language is developed to interact with the database. It is successfully implemented in all DBMS for the user convenience. [8][9]

Industries applications are developed using the sql and lacks to provide a flexible environment for the user. Fuzzy logic is the improvement of binary logic and widely used to solve the complex problem. Many applications have implemented fuzzy sql to sort out the problems related to the interaction with different languages.[10][11]
Intuitionistic fuzzy logic further enhances the fuzzy logic using its intuitionistic approach to the problem. The research is to develop sql answering system based on intuitionistic fuzzy. The base of the system is pictorially depicted in the figure 1.

\subsection{User input}

The user input will be from different region and language. Users are allowed to input any kind of language and expressions for the interaction with the system.[12][13]

\subsection{Third party translator}

Many translation applications are available in the internet and google api's are very popular for the distributed database application. The preciseness of the application is upto the maximum so it can be easily implemented in database application.[15]

\subsection{Fuzzifier}

The process of fuzzification is very important for the research. The translation process may not produce the optimum result so this stage will pre-process the input and check whether the translated input is in correct form.

\subsection{Query optimization}

The stage will optimize the pre-processed input and make it ready for the acceptance of the server. The server will generate the result after the acceptance of the query.

The further part of the paper will explain the review of literature and experiment results.

\section{REVIEW OF LITERATURE}

Marlene Goncalves et. al., proposed a research in flexible sql. The work has defined some extensions in sqlf as sqlf3, allows the use of fuzzy implemented functions. The research has sorted out the major pitfalls in sql.[1]

Yanhui Lv et. al., have proposed a framework based on fuzzy ontology generation based on $n-$ ary descriptions. The fuzzy ontology framework can be mapped to fuzzy description logic and fuzzy defined functions.[2] 


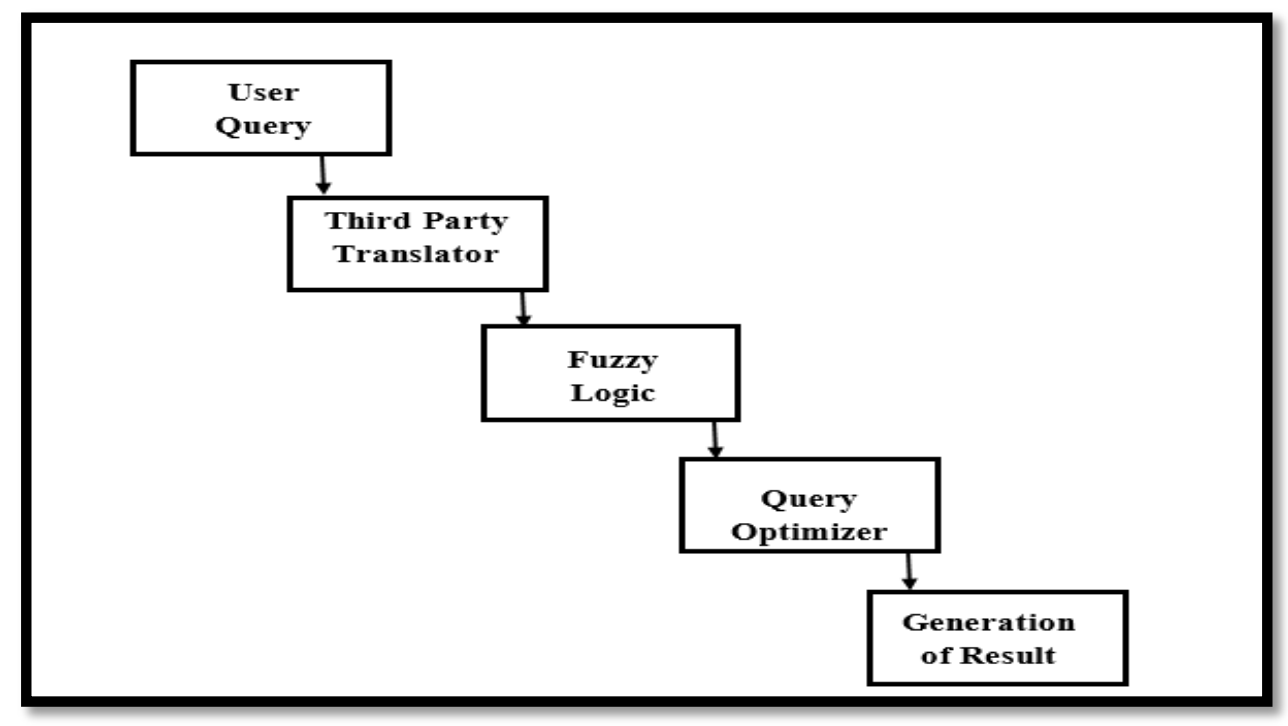

Fig 1: Answering system based on Intuitionistic fuzzy

Shyi - ming et. al., have presented a method to handle the vagueness found in the user queries. The research employed fuzzy logic to remove the impreciseness in the query submitted by the inexperienced users. The proposed method provide flexible and friendly environment.[3]

Ke Xiao - hua proposed an automatic translation system based on semantic similarities and fuzzy neartude. The system can identify the semantic characteristics and yield scores that corresponds with human judgements of translation quality.[4]

Zheng qin developed a method of mathematical translation of expressive preferences of people by eliminating the traditional preferences. The research employed fuzzy logic to find the people's mood. They have done a test to get the respond's expressive preferences. [5]

Amit Garg et.al., proposed a framework for fuzzy sql. They have generalized logical condition for the "where" part of sql. They have deployed application layer over sql to make a crisp query from queries with linguistic hedges.[6]

Ulli waltinger et.al., have made a question - answering system over semi - structured industry data. The proposed method allows user to query in their natural way, then the system adjusts the query and generates the result according to the query.[7]

Abhijeet R. Raipurkar et.al., proposed a method for query optimization using fuzzy logic. The system adjusts the user query using fuzzy logic and make the process more easier to access for the users.[8]

\section{RESULT AND DISCUSSION}

The research is developed for the purpose of the business to organize the database in an easier way for the users. The work has employed intuitionistic fuzzy for the pre-processing of queries sent by the user from different regions. As shownin the figure 2, users from different regions are asked to interact with the system. User - friendly environment has been set for the users, they can use their natural and flexible language for interaction.Third party translators are used to translate the queries. Google API's are used for the research and found more flexible for the translation of queries. The user query from different locations shown in fig 2, indicates the distributed nature of the model. The model has the ability to work in network environment. In the present scenario, organizations have onshore and offshore business and interact with the wide variety of users. Organization need a model to reduce the risk of communication with the customer.

Google has introduced a feature of publishing the concept of development tools and assist programmers to develop a research or application. Researchers find lot of google API's in internet and customize it according to their purpose of development. In this work, the user queries will be translated through the google API and translated queries will be adjusted by the fuzzifier because translators will produce a generalized translation of the user query. The fuzzifier will modify the translated query into specific query and forward to the query optimizer. The query optimizer will optimize the query according to the syntax and semantics of sql and submit to the server and return the response from the server to the user through fuzzifier and translator.

Intuitionistic fuzzy receives the translated queries from the previous stage and pre-process the query. During the translation, some of the words are not in proper format or some vagueness will be found in the sentences and these kind of impreciseness and vagueness will be removed and adjustment will be made on the sentences.

Query optimizer employed in the research will normalize the input sent by the fuzzifier. Usually the fuzzifier will preprocess the user input even though the input will not be perfect for submission to the server/database.

Select, Input, Delete and Triggers are executed in the distributed environment. The following table 1 shows that sql with intuitionistic fuzzy performance is better than the other two formats. Sql with fuzy will perform well in the standalone / centralized environment but the performance will not be good in the distributed environment. 


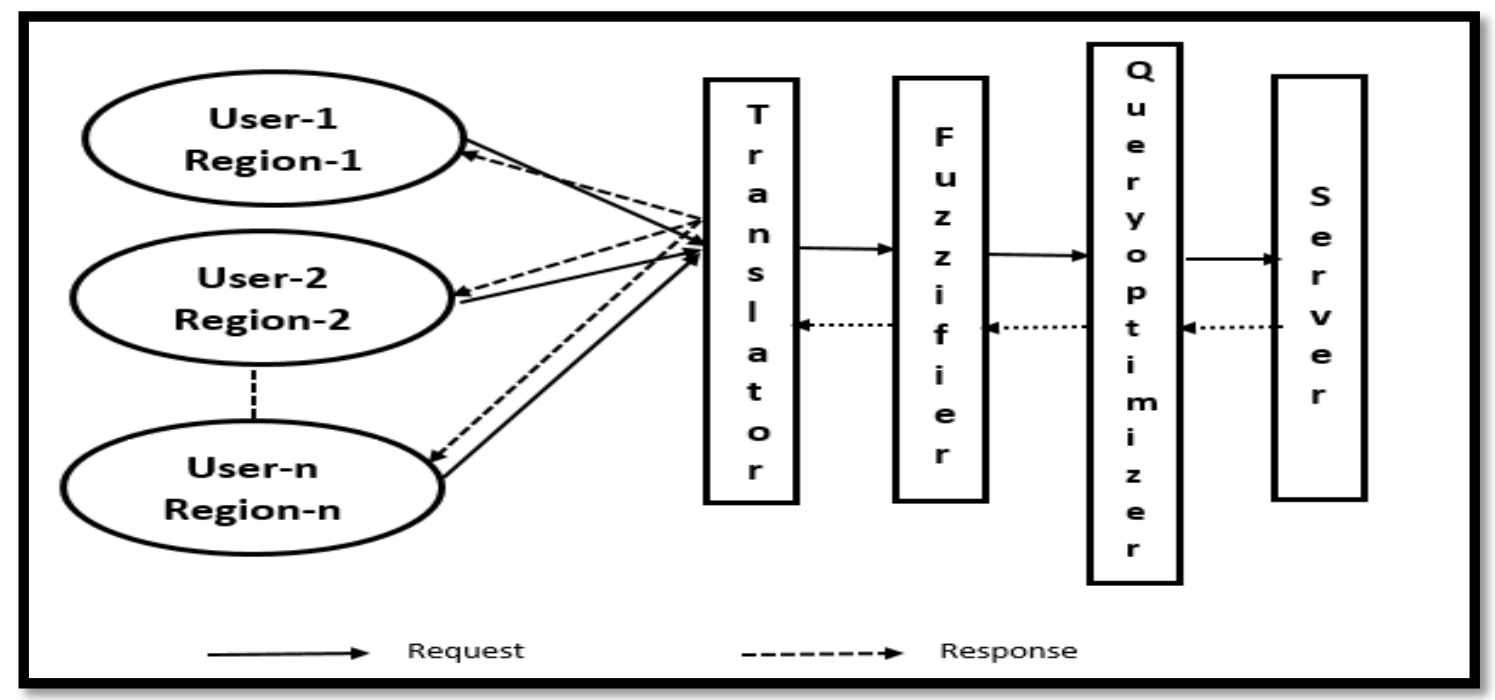

Fig 2: User interaction with the system

Table 1. Comparison of results of the experiment

\begin{tabular}{|c|c|c|c|}
\hline $\begin{array}{c}\text { Query / } \\
\text { Method }\end{array}$ & $\begin{array}{c}\text { SQL } \\
(\mathrm{Sec})\end{array}$ & $\begin{array}{c}\text { FSQL } \\
(\mathrm{Sec})\end{array}$ & $\begin{array}{c}\text { IFSQL } \\
(\mathrm{Sec})\end{array}$ \\
\hline Select & Error & $\mathbf{2 0 0}$ & $\mathbf{1 5 0}$ \\
\hline Insert & Error & Error & $\mathbf{1 2 0}$ \\
\hline Delete & Error & $\mathbf{1 5 0}$ & $\mathbf{1 0 0}$ \\
\hline Triggers & Error & Error & $\mathbf{1 5 0}$ \\
\hline Others & Error & Error & $\mathbf{1 3 0}$ \\
\hline
\end{tabular}

Table 2.Performance of SQL

\begin{tabular}{|l|c|c|}
\hline $\begin{array}{c}\text { Query / } \\
\text { Method }\end{array}$ & $\begin{array}{c}\text { Response } \\
\text { Time } \\
\text { (Sec) }\end{array}$ & $\begin{array}{c}\text { Space } \\
\text { Complexity } \\
\text { (KB) }\end{array}$ \\
\hline Select & 0.25 & 120 \\
\hline Insert & 0.27 & 125 \\
\hline Delete & 0.28 & 95 \\
\hline Triggers & 0.29 & 120 \\
\hline Others & 0.32 & 105 \\
\hline
\end{tabular}

Table 2 shows the performance of the sql in the answering system. The values shown in the table indicates its time and space complexity to execute a query submitted by the user to the answering system. Same queries are executed with 3 types of sql and space allocation will be different for normal, fuzzy and intuitionistic fuzzy methods.
Table 3.Performance of FSQL

\begin{tabular}{|c|c|c|}
\hline $\begin{array}{c}\text { Query / } \\
\text { Method }\end{array}$ & $\begin{array}{c}\text { Response } \\
\text { Time } \\
(\text { Sec })\end{array}$ & $\begin{array}{c}\text { Space } \\
\text { Complexity } \\
(\text { KB })\end{array}$ \\
\hline Select & $\mathbf{0 . 2 1}$ & 95 \\
\hline Insert & $\mathbf{0 . 2 3}$ & 102 \\
\hline Delete & $\mathbf{0 . 2 9}$ & 110 \\
\hline Triggers & $\mathbf{0 . 3 1}$ & 120 \\
\hline Others & $\mathbf{0 . 2 1}$ & 110 \\
\hline
\end{tabular}

Table 3 shows the performance of Fsql towards the user queries submitted to the Answering system. The results show improvement in the time and space complexity because of the nature of the fuzzy logic.

Table 4.Performance of IFSQL

\begin{tabular}{|l|c|c|}
\hline $\begin{array}{c}\text { Query / } \\
\text { Method }\end{array}$ & $\begin{array}{c}\text { Response } \\
\text { Time } \\
(\text { Sec })\end{array}$ & $\begin{array}{c}\text { Space } \\
\text { Complexity } \\
(\text { KB })\end{array}$ \\
\hline Select & $\mathbf{0 . 1 7}$ & $\mathbf{8 5}$ \\
\hline Insert & $\mathbf{0 . 1 9}$ & 79 \\
\hline Delete & $\mathbf{0 . 1 7}$ & $\mathbf{9 1}$ \\
\hline Triggers & $\mathbf{0 . 1 6}$ & 100 \\
\hline Others & $\mathbf{0 . 1 8}$ & 100 \\
\hline
\end{tabular}

Table 4 shows the performance of IFSQL and results show the better performance of the proposed research and following figure 3 and 4 shows the graphical representation of the time and space complexity. The curve at the bottom show the IFSQL performance. It takes less space and time to execute the query. 


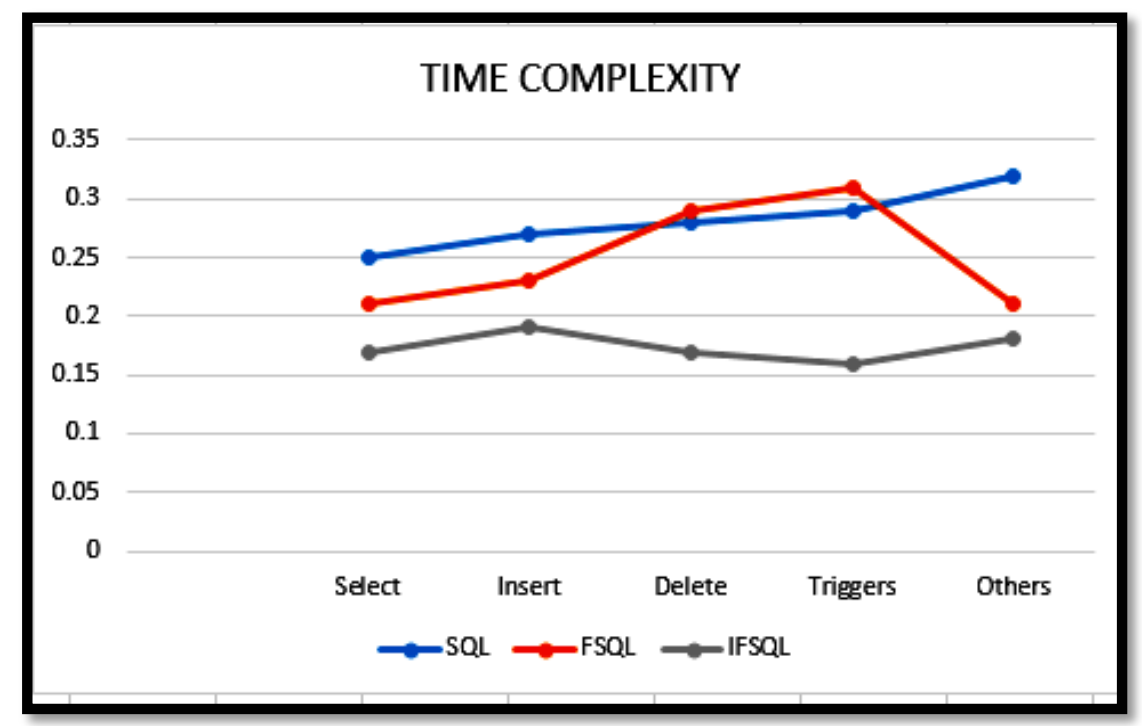

Figure 3 Graphical representation of Time complexity

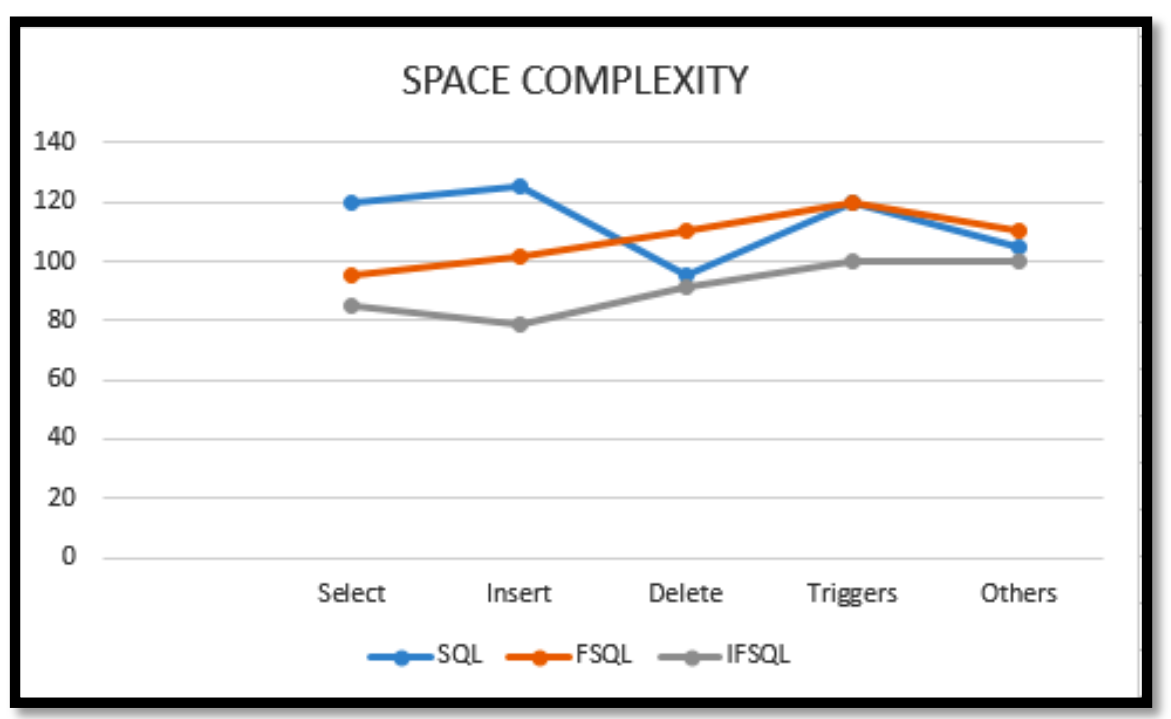

Figure 3 Graphical representation of Time complexity

\section{CONCLUSION}

Answering system should be effective for the industries to expand their business all over the world. Database interactivity should be streamlined with the activities corresponding with the users input. The research has developed the model with the intuition to answer the user queries and provide flexible environment for the users interact with the system. The results show the better performance of the proposed research with different types of queries. Fuzzy and intuitionistic fuzzy are the machine learning methods can be employed to automate a process and generate effective results. The future scope of the research is to build a model for the symbolic representation of the user queries. User might not express queries in a proper format so symbols or general short codes for the expression can be used to interact with the system.

\section{REFERENCES}

[1] Marlene Goncalves and Leonid Tineo, "SQLf3: An extension of SQLF with SQL3 features", IEEE International fuzzy systems conference, PP. $477-480$.

[2] Yanhui Lv, Z.M.Ma and Fu zhang, “ A fuzzy ontology generation framework from relational schema", Fifth international conference on fuzzy systems and knowledge discovery, PP. $435-439$.

[3] Shyi- Ming and Woei - Tzy Jong," Fuzzy query translation for relational database systems", IEEE transactions on systems, man and cybernetics - part B: Cybernetics, Vol. 27, No.4, August 1997.

[4] Ke Xiao - Hua, "An automatic translation evaluation system based on semantic similairities and fuzzy neartude", 2009 International conference on environmental science and information application technology, pp. $583-586$. 
[5] Zheng Qin and Qing - Chen Shang, "Eliminate people's expressive preference in the mood of fuzzy linguistics", Krakow, Poland, May 25-27, 2008. pp.1021- 1026.

[6] Amit Garg and Rahul Rishi, "Querying capability enhancement in database using fuzzy logic", Global journal of computer science and technology, Vol.12,Iss.6, 2012.

[7] Ulli waltinger, Dan Tecuci, Mihaela Olteanu, Vlad Mocanu and Sean Sullivan, " USI Answers: Natural Language Question Answering over (Semi-) Structured Industry Data", Proceedings of the Twenty - Fifth applications of artificial intelligence conference, pp.1471 -1478 .

[8] Abhijeet R.Raipurkar and G.R. Bamnote," Fuzzy logic based query optimization in distributed database", International journal of innovative research in computer and communication engineering", Vol.1, Iss.2, April 2013. pp. $422-426$.

[9] C.T.Yu, K.C.Guh, W.Zhang, M. Templeton, D.Brill and A.L.P. Chen, " An integrated algorithm for distributed query processing”, IFIP Conference, Amsterdam, 1987.
[10] J.K.T. Huang, “ Query optimization in Distributed databases", Conference of information and decision systems, MIT.1982.

[11] M.S.Chen and P.S.Yu, “ A graph theoretical approach to determine a join reducer sequences in Distributed query processing", Vol.5:3, pp.534-542, 1993.

[12] Jyothsna Cherapanamjeri, Lavanya Lingareddy, Himabindu. K, "Keyword based question answering system in natural language interface to database", IJARCET, Vol.3, Iss.12, December 2014.

[13] N. Ramireddy, Sivaji Bandyopadhya, “ Dialog based question answering system in Telugu," EACL workshopon Multilingual Question Answering., 2006.

[14] Dhanshri patil, Abhijeet chopade, Pankaj bhambare, sanket deshmukh and aniket tetame," A proposed automatic answering system for natural language questions”, IJEC, Vol.4,Iss.4, April 2015. Pp. 1131011312.

[15] S.R.Balasundaram and B.Ramadoss, "SMS for question - answering in the m-learning scenario, journal of computer science 3(2), pp. 119 - 121, 2007 